\title{
P-T induced re-distribution of hydrogen defects in nominally anhydrous minerals
}

\author{
YAN YANG \\ Zhejiang University \\ Presenting Author: yanyang2005@zju.edu.cn
}

Knowledge of hydrogen defects in nominally anhydrous minerals (NAMs) is fundamental to understand physicalchemical processes in the deep Earth. Many cases indicate that IR spectral features of hydrogen defects in the same mineral from different geological settings are different, and spectra features of hydrogen defects in some synthetic samples cannot match those in natural samples. Furthermore, different hydrogen defects have different behaviors, such as previously reported sitespecific IR absorption coefficients, site-specific diffusivities and site-specific water effects on physical properties of the host mineral. Therefore, it is important to clarify whether hydrogen defects can be preserved in samples quenched from P-T conditions of the deep Earth.

Here, we display some cases for hydrogen defects in feldspar, olivine, clinopyroxene, orthopyroxene, wadsleyite, ringwoodite, rutile. Their IR spectra at high temperatures indicate the possible re-distribution of hydrogen defects. For hydrogen defects in feldspar and rutile, the atomic site for hydrogen in the crystal structure changes with increasing temperature, and the redistribution is reversible with no new hydrogen defects appearing. In contrast, for hydrogen defects in olivine, clinopyroxene, orthopyroxene, wadsleyite and ringwoodite, the re-distribution is irreversible with some new hydrogen defects occurring at the cost of dehydration. These results are enlightening for interpreting spectra features, and evaluating water impacts on physical-chemical processes in the deep Earth. 